\title{
Japanese Classification of Gastric Carcinoma - 2nd English Edition - Response assessment of chemotherapy and radiotherapy for gastric carcinoma: clinical criteria
}

\author{
Japanese Gastric Cancer Association \\ Association office, Department of Digestive Surgery, Kyoto Prefectural University of Medicine, Kawaramachi, Kamigyo-ku, Kyoto 602-0841, \\ Japan
}

\section{Foreword}

The World Health Organization (WHO) criteria for the assessment of response to nonsurgical cancer treatment (Reporting Response in the WHO Handbook, 1979) have been proven to provide a durable international standard. These criteria alone, however, insufficiently assess the results of treatment in the setting of gastric cancer.

Recognizing this problem, and adhering to the principles encompassed by the WHO criteria, the Japanese Research Society for Gastric Cancer (JRSGC), in 1985, established new assessment criteria for gastric cancer, including quantitative criteria for assessing primary tumor responses. Two aspects of these criteria deserve particular emphasis: (1) the use of X-ray and endoscopic findings for the assessment of primary gastric lesions and (2) new methodology specific to diffusely infiltrating tumors. Under this JRSGC system, primary lesions were broadly classified as: (a) measurable, (b) evaluable but not measurable, and (c) diffusely infiltrating.

These criteria were revised in 1993, concurrently with the General rules for gastric cancer study. Detailed descriptions of these criteria were published in English, for an international readership, in 1995 [1]. During the 6 years since the 1993 revision, several investigators have confirmed that the overall response according to these criteria correlated with survival time. After the establishment of the Japanese Gastric Cancer Association, reorganized from the JRSGC in 1997, these Japanese response criteria were further refined [2]. These criteria,

\footnotetext{
The entire contents of this article can be downloaded as a PDF file on the website of the Japanese Gastric Cancer Association (http://1surg.kpu-m.ac.jp/jsgcr.htm), together with the first part of the Japanese Classification of Gastric Carcinoma. Reprints are not available.
}

faithfully translated from the Japanese edition into English, are presented here.

\section{Introduction}

The clinical criteria for assessment of response to nonsurgical treatment of gastric cancer can be categorized under six headings: patient status at the start of therapy, description of therapy, clinical therapeutic efficacy, duration of survival, adverse drug reactions, and "other features."

\section{Patient's status at the start of therapy}

(1) Performance status Grade 0-4 (According to the "WHO criteria").

(2) Concurrent disease(s)

Describe the presence and name(s) of concurrent disease(s) that may influence the outcome of chemotherapy or radiation therapy.

(3) Description of lesion(s)

Describe the presence of previous treatment and recurrence as well as the "stage." For measurable lesion(s), describe the size(s) and the method used for the measurement.

\section{Description of therapy}

(1) Chemotherapy

(1) Name of the drug, (2) route of administration, (3) interval of administration, (4) dosage or daily dose, (5) total number of administrations, (6) total dose, (7) period of administration, (8) reason for discontinuation, (9) presence and name(s) of ad- 
verse drug reaction(s), and (10) presence and name(s) of other therapy or therapies that may influence the outcome of chemotherapy.

(2) Radiation therapy

(1) Purpose of irradiation (curable, incurable, or nosotropic), (2) apparatus, (3) name of irradiation, (4) energy, (5) conditions of irradiation, (6) collimation, (7) size of irradiated field, (8) name of irradiated area, (9) dose per exposure, (10) total exposure dose, (11) fractionation, (12) irradiation period.

\section{Clinical therapeutic efficacy}

(1) General objective/subjective findings (Table 1)

(2) Tumor response

1. Methods of measurement

a. For gastric lesion(s), describe the tumor response, morphological change, and efficacy, evaluated by $\mathrm{X}$-ray and/or endoscopic examinations.

b. For metastatic lesion(s), measure the lesion(s) according to the "Efficacy criteria for solid tumors" (these criteria closely match the WHO criteria) published by the Japan Society for Cancer Therapy and judge the efficacy.

2. Evaluation method for gastric lesions

a. Measurable lesions (a-lesions)

(i) Two-dimensionally measurable lesions Calculate the product of the longest diameter and the longest rectangular diameter.

Response rate $=$

(product calculated before therapy)

$\frac{- \text { (product calculated after therapy) }}{\text { (product calculated before therapy) }} \times 100 \%$

Table 1. Objective/subjective findings

\begin{tabular}{|c|c|c|c|}
\hline General & \multicolumn{3}{|c|}{$\begin{array}{c}\text { Maximum efficacy achieved by } \\
\text { therapy }\end{array}$} \\
\hline . Pain & \multicolumn{3}{|c|}{ Improved-unchanged-exacerbated } \\
\hline 2. Anorexia & \multicolumn{3}{|c|}{ Improved-unchanged-exacerbated } \\
\hline 3. Nausea-vomiting & \multicolumn{3}{|c|}{ Improved-unchanged-exacerbated } \\
\hline 4. Other ( ) & \multicolumn{3}{|c|}{ Improved-unchanged-exacerbated } \\
\hline Reference: & & & \\
\hline 5. Body weight change ${ }^{a}$ & & $\mathrm{~kg} \rightarrow$ & $\mathrm{kg})$ \\
\hline 6. Perforr & & $\rightarrow$ & ) \\
\hline 7. Tumol & value ( & $\rightarrow$ & ) \\
\hline 8. QOL score ${ }^{\mathrm{b}}$ & ( & $\rightarrow$ & ) \\
\hline
\end{tabular}

QOL, Quality of life

${ }^{a}$ Unevaluable in patients with pleural effusion/ascites

${ }^{\mathrm{b}}$ Questionnaire used (see reference [3]) (ii) One-dimensional measurable lesions

Response rate $=$

(longest diameter before therapy)

$\frac{- \text { (longest diameter after therapy) }}{\text { (longest diameter before therapy) }} \times 100 \%$

(longest diameter before thera

b. Evaluable but not measurable lesions (b-lesions)

(i) Describe changes in protruded lesions as follows:

Progression, no change, regression: flattening, or disappearance

(ii) Describe changes in excavated lesions as follows:

- Raised margin: progression, no change, regression: flattening, or disappearance

- Crater: progression, no change, regression: flattening, disappearance

c. Diffusely infiltrating lesions (c-lesions)

In diffusely infiltrating tumors (type 4), tumor volume cannot be assessed by X-ray or endoscopy. Treatment response may be evaluated by expansion of the gastric lumen. For all assessments, comparison must be made with the status of the lesion before treatment.

In principle, calculate the square of the lesion shown with standing barium X-ray examination, and calculate the enlargement rate. Use the same position and the same volume of barium for comparison.

Enlargement rate $=$

(product calculated before therapy)

$\frac{- \text { (product calculated after therapy) }}{\text { (product calculated before therapy) }} \times 100 \%$

3. Definition of response in gastric lesions

In patients with complete response (CR), partial response (PR), and no change (NC), no new lesions should appear within 4 weeks and each defined condition should last for more than 4 weeks.

\section{Complete response (CR)}

Disappearance of all tumoral lesions and no diagnosis of any cancers.

\section{Partial response (PR)}

a-lesions

At least a $50 \%$ decrease in total tumor size in two-dimensional measurable lesions and at least a $30 \%$ decrease in total tumor size in onedimensional measurable lesions.

b-lesions

Dramatic regression: flattening on X-ray/ endoscopic examinations, which roughly corre- 


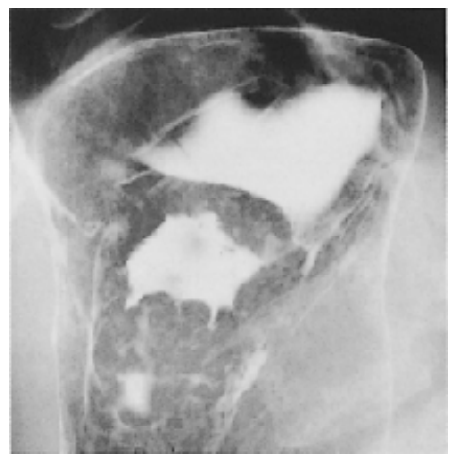

Pretreatment (X-ray): A protruded lesion with a crater can be seen in the center of the posterior wall of the upper third of the stomach. Type $2^{\prime}$ tumor, 8 $\times 6 \mathrm{~cm}$ in area

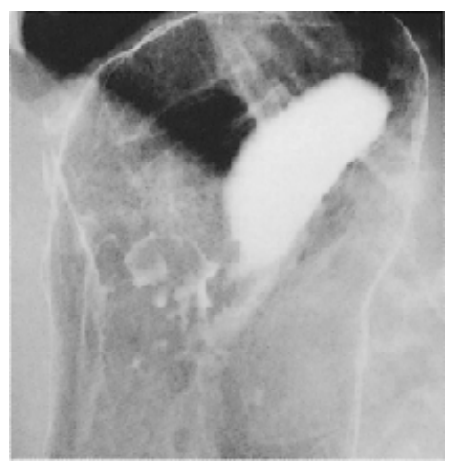

6 weeks after treatment: Flattening of both the crater and the margin. $5 \times 4.5 \mathrm{~cm}$ in area

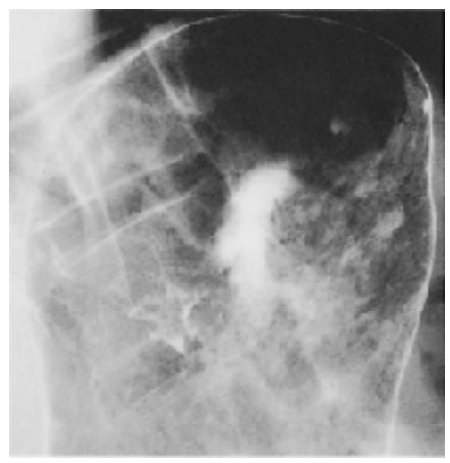

11 weeks after treatment: The previously raised margin has flattened. Type 0 IIc, $3.5 \times$ $3.5 \mathrm{~cm}$ in size

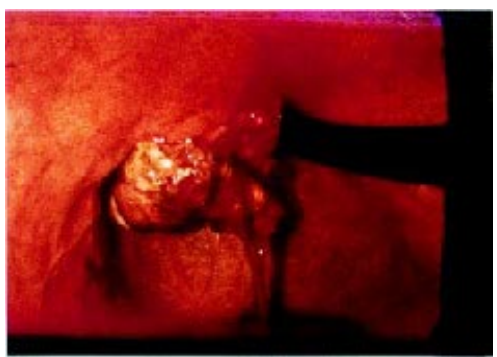

Pretreatment (endoscopic findings): An irregularly shaped tumor with hemorrhage can be found in the posterior wall of the upper third of the stomach

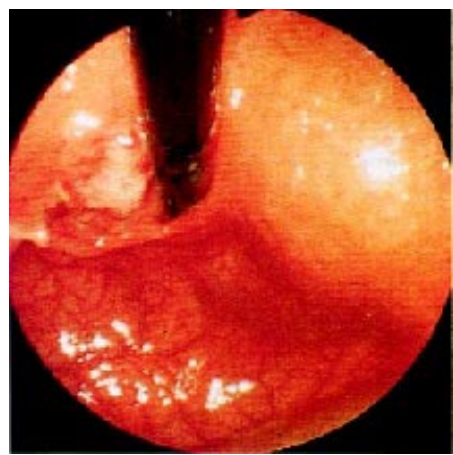

5 weeks after treatment: The lesion has regressed and flattened

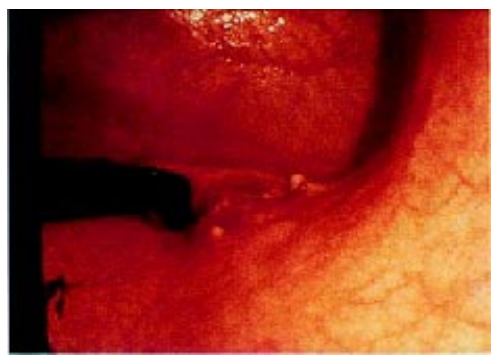

11 weeks after treatment: The tumor continues to regress

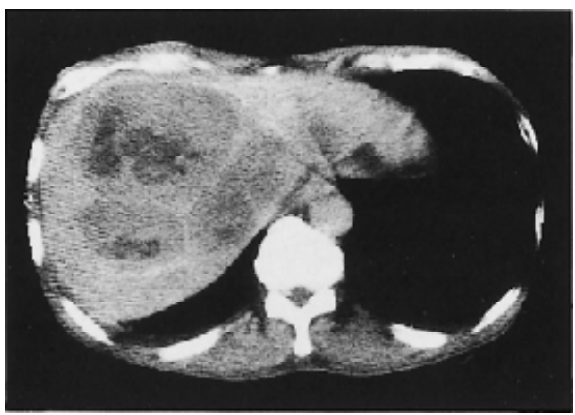

Pretreatment (CT): Metastasis to the liver. Numerous, low-density, necrotic masses can be found throughout the right lobe of the liver

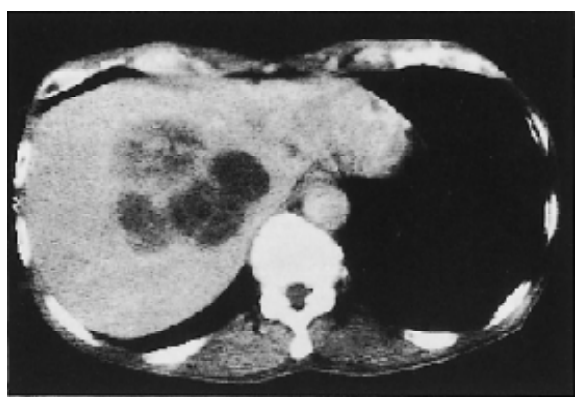

5 weeks after treatment: The masses in the right lobe have regressed $(56 \%)$

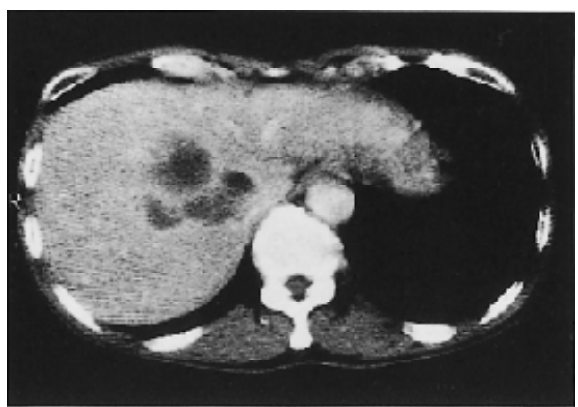

10 weeks after treatment: The masses in the right lobe continue to regress $(78 \%)$

Fig. 1. Chemotherapeutic response in a patient with nonoperated gastric a-lesion cancer with hepatic cancerous metastasic lesions assessed by X-ray, endoscopic findings, and computed tomography (CT) scans. This case was definitely evaluated as a partial response (PR) by the PR duration of more than 6 weeks, assessed at 5 and 11 weeks after treatment. See text for definition of "a-lesion" 


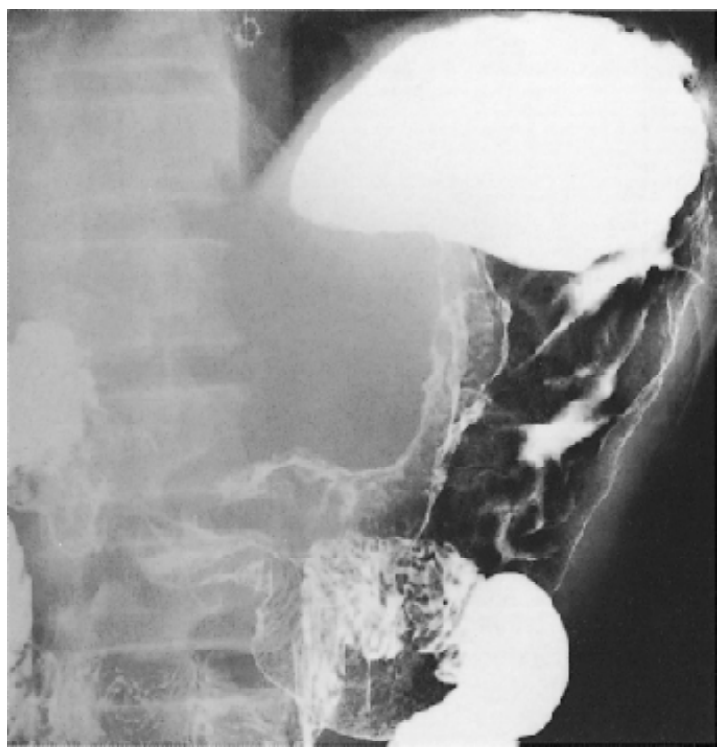

Pretreatment (X-ray): An excavated lesion with a raised margin extending from the gastric body to the antrum
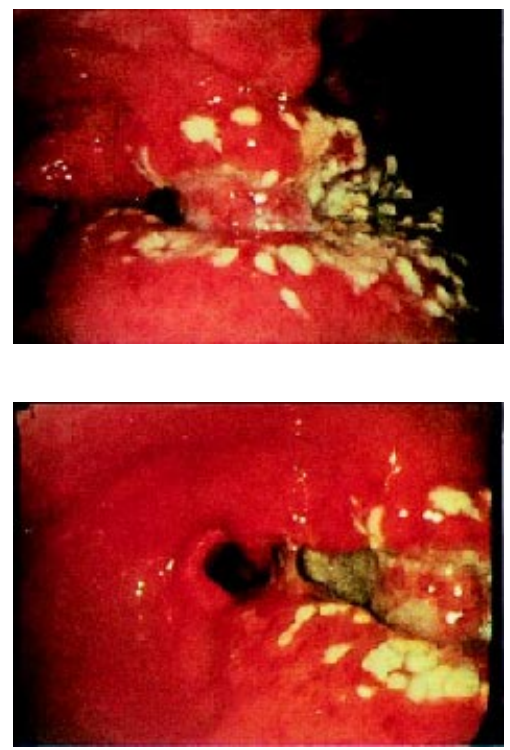

Pretreatment (endoscopic findings): An excavated lesion with a raised margin and surrounding white plaques extending from the gastric body to the antrum

Fig. 2. Chemotherapeutic response in a patient with nonoperated gastric b-lesion cancer assessed by X-ray and endoscopic findings. This case was definitely evaluated as PR for the primary b-lesion by the PR duration of more than 20 weeks assessed at 41 and 61 weeks after treatment. See text for definition of "b-lesion"

sponds to at least a $50 \%$ decrease in tumor size.

c-lesions

In diffusely infiltrating lesions, at least $50 \%$ enlargement of the gastric lumen in the area of the lesions by X-ray examination.

\section{No change (NC)}

Insufficient decrease in a- and b-lesions and in sufficient change in c-lesions on X-ray/endoscopic findings to qualify for a PR, or findings that are the same as baseline.

\section{Progressive disease (PD)}

Exacerbation of tumor size or X-ray/endoscopic findings (at least a $25 \%$ increase in a-lesions) or the appearance of new lesions.

\section{Minor response (MR)}

Response is separately described in each of the following cases:

- PR for less than 4 weeks

- In a- and b-lesions, less than $50 \%$ but more than $25 \%$ decrease in tumor size; in c-lesions enlargement of the gastric lumen lasting for 4 weeks at least but of insufficient extent to qualify from PR
4. Overall response

Patients with $\mathrm{CR}$ or PR in gastric or metastatic lesions are defined as responders for the calculation of overall response. For the total treated population, eligible cases, complete cases, or the "Full analysis set [4]" should be described.

5. Overall response in patients with multiple lesions Evaluate individual response in each organ. Overall response is defined as follows:

$\mathrm{CR}, \mathrm{CR}$ in all organs

$\mathrm{PR}$, total number of $\mathrm{CR}$ and $\mathrm{PR}$ is more than that of NC

$\mathrm{NC}$, number of $\mathrm{NC}$ exceeds the total number of $\mathrm{CR}$ and $\mathrm{PR}$

$\mathrm{PD}$, at least one PD in any organ, or the appearance of new lesions.

6. Duration of response

The following dates should be recorded for measurement of duration of response:

A, date of initiation of the treatment

$\mathrm{B}$, date when obvious tumor regression is first observed

$\mathrm{C}$, date when at least $50 \%$ decrease is first observed 


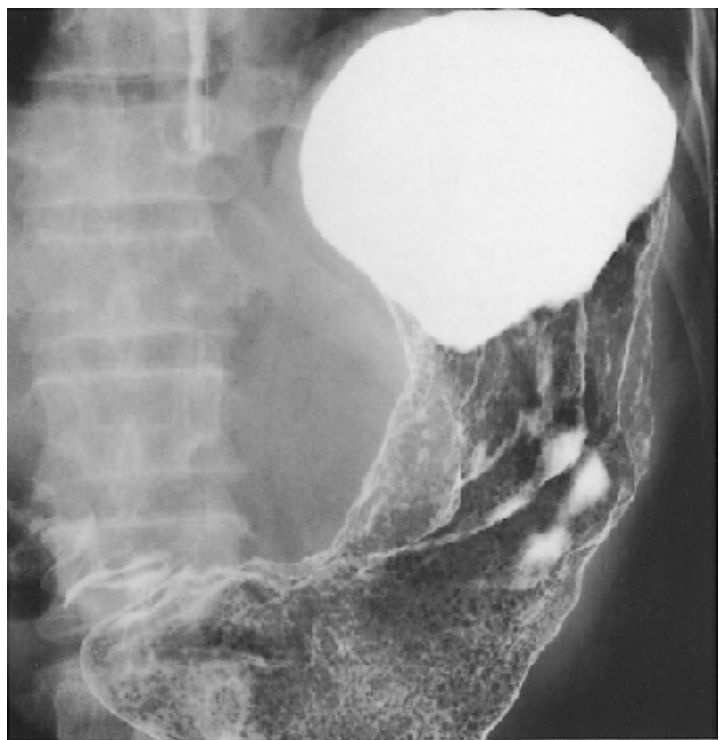

41 weeks after treatment: The raised margin and crater have flattened

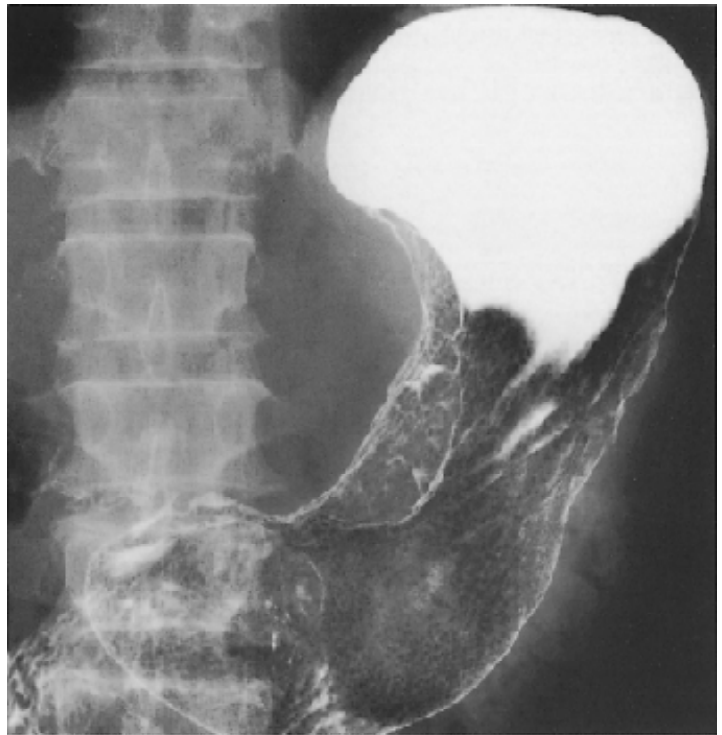

62 weeks after treatment: The raised margin and crater remain flat

Fig. 2. continued

$\mathrm{D}$, date when disappearance of all tumors is first observed

$\mathrm{E}$, date when regrowth of shrunken lesions or growth of new lesions is first observed.
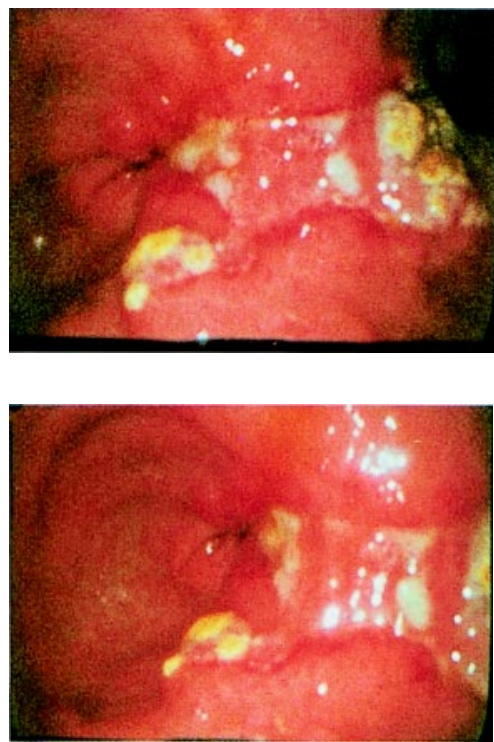

41 weeks after treatment: The raised margin, crater, and white plaques are disappearing
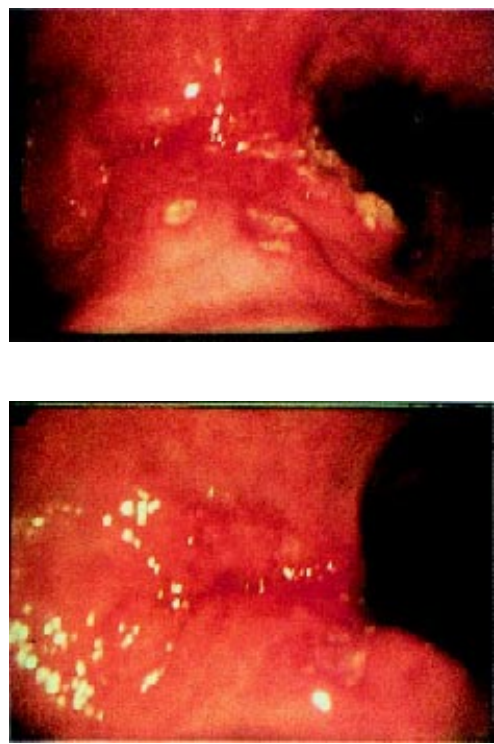

61 weeks after treatment: The raised margin remains flat. The crater is smaller
Duration of response is defined as follows:

Duration of $\mathrm{CR}=\mathrm{D}$ minus $\mathrm{E}$

Duration of $\mathrm{PR}=\mathrm{C}$ minus $\mathrm{E}$

Duration of overall response $=\mathrm{A}$ minus $\mathrm{E}$.

* Only CR achieved by this treatment is included in the calculation of the response. Results of cytodiagnosis are not necessary for the evaluation of the therapeutic response; however, when possible, the results should be described separately. 


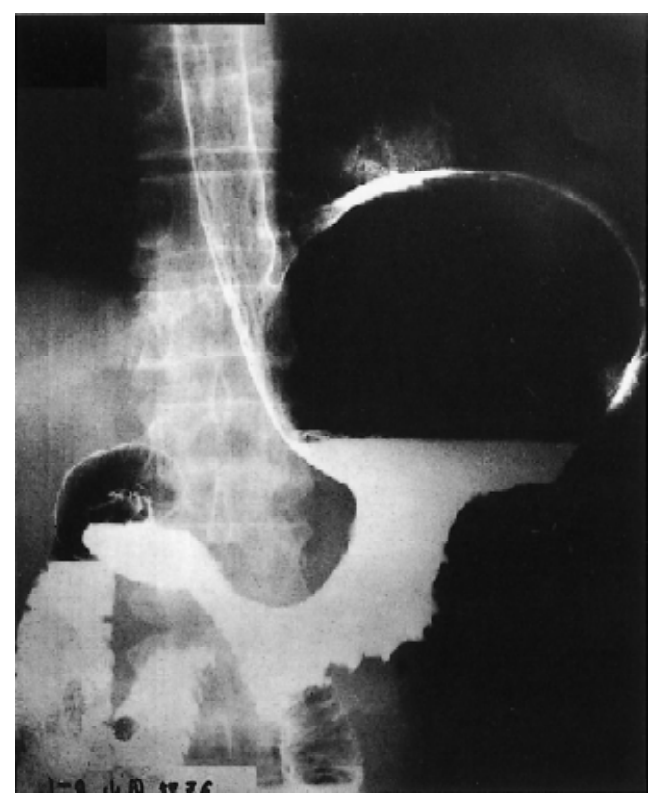

Pretreatment, standing A.P. barium study: From body to pylorus, the stomach is narrowed and shrunken. In this patient, extensive invasion of the pancreas and mesocolon prevented surgical removal

Fig. 3. Chemotherapeutic response in a patient with nonoperated gastric c-lesion cancer, assessed by X-ray. The border between the cancerous lesion and normal area must be defined on the X-ray film. The dividing line can be defined as extending from point $A$ on the lesser curvature to point $B$ on the greater curvature line. The area of the gastric lumen below the dividing line was evaluated in the primary X-ray. The reference line was determined from point $C$ at His' angle to point $D$ at the intersection of a line from point $C$ to the

7. Evaluation of response in patients with pleural effusion/ascites*

$\mathrm{CR}$, complete disappearance of pleural effusion/ ascites for at least 4 weeks

PR, dramatic decrease in pleural effusion/ascites for at least 4 weeks

No response, failure to meet any of the above criteria.

Actual clinical examples of a-, b-, and c-lesions are shown in Figs. 1-3, respectively.

\section{Duration of survival}

Duration of survival is measured from the date of the beginning of treatment until the date of the patient's death (in weeks). For the total treated population,

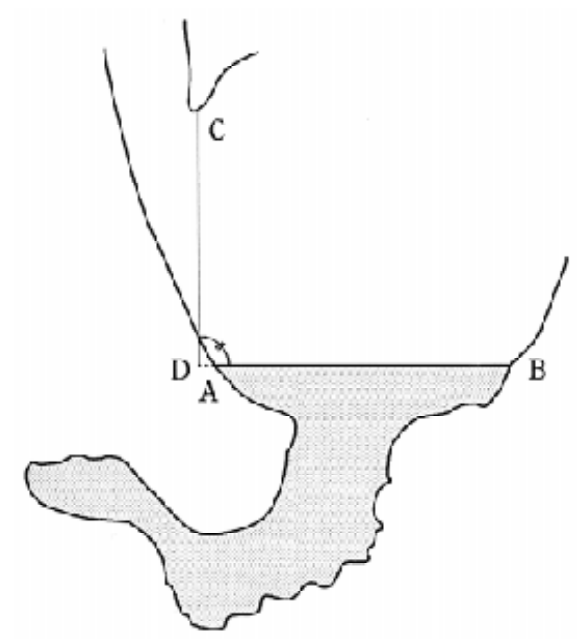

The gray area must be calculated according to the following factors:

Dividing line: point A to point B

Reference line: point $\mathrm{C}$ to point $\mathrm{D}$

Gray area: $56.06 \mathrm{~cm}^{2}$

extended dividing line on the pretreatment X-ray. For evaluation of the post-chemotherapeutic effect, the dividing points and the dividing line were defined from the reference line of equal length and the equal angle between the reference line and dividing line on the primary X-ray. This case was definitely evaluated as PR for the primary c-lesion by the PR duration of 26 weeks, as assessed at 12 and 38 weeks after treatment. See text for definition of c-lesion. A.P., Anterior-posterior

eligible cases, complete cases, and a "full analysis set [4]" should be described.

(1) $50 \%$ Survival duration

This is the duration in which the cumulative survival rate in the total treated population becomes $50 \%$.

(2) Survival rates (1- to 5-year)

Calculate 1- to 5-year cumulative survival rates in the total treated population (by the Kaplan-Meier method or the life-table method).

\section{Adverse drug reactions}

Describe the toxicity and severity and date of onset and duration, according to the Revised common toxicity criteria (CTC) version 2.0. 


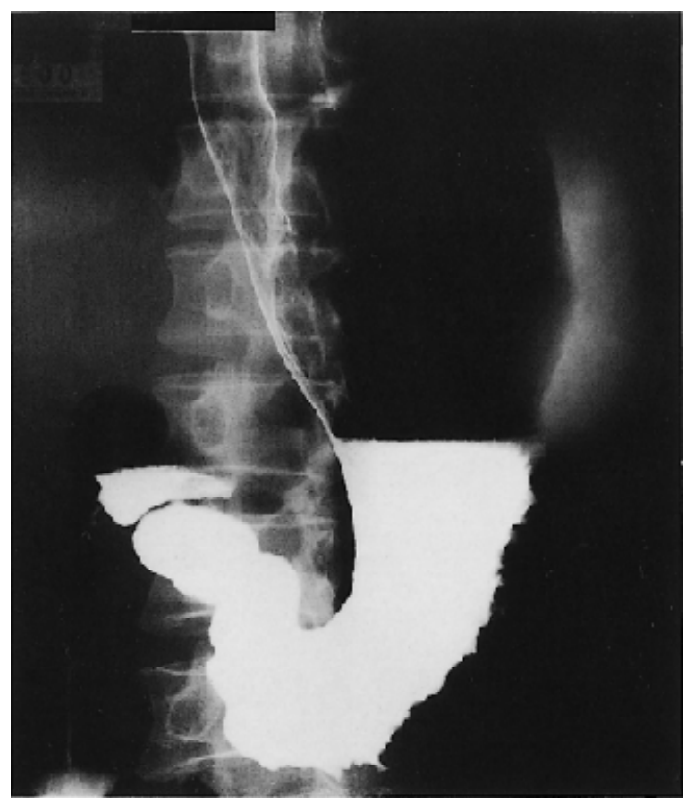

12 weeks after treatment, standing A.P. barium study: Gastric capacity and lumen diameter have clearly increased

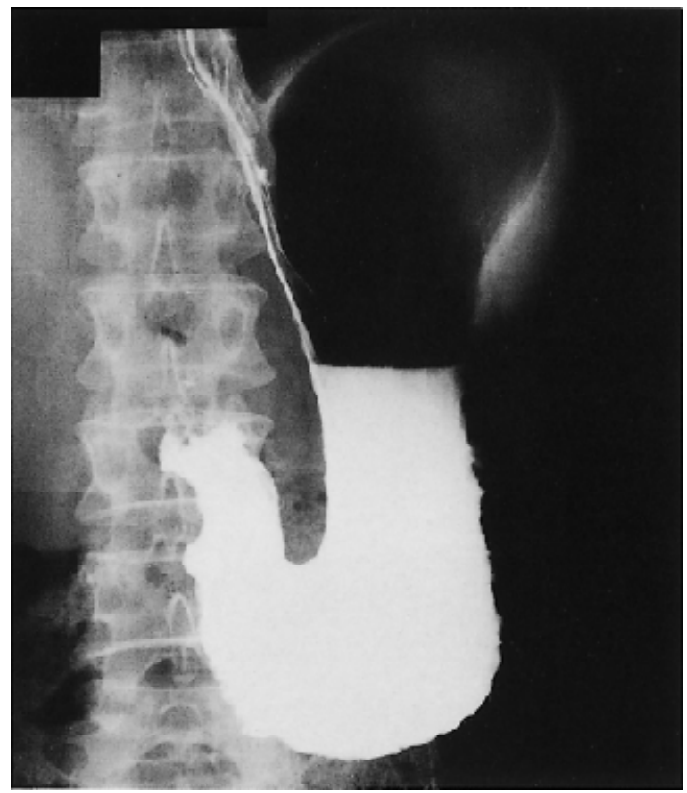

38 weeks after treatment, standing A.P. barium study: While the dividing line is no longer distinguishable, the deformed stomach and the hardened gastric wall imply residual malignancy

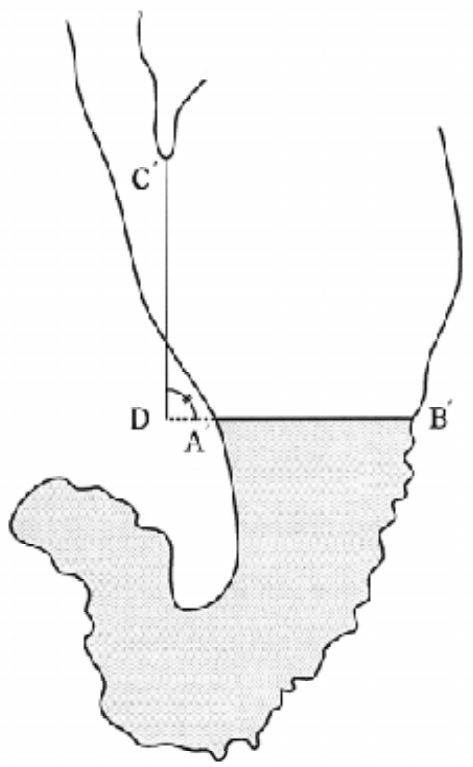

The gray area must be calculated according to the following factors: Dividing line: point A' to point B' Reference line: point $\mathrm{C}$ to point $\mathrm{D}$ Gray area: $111.60 \mathrm{~cm}^{2} ; 99.07 \%$ increased

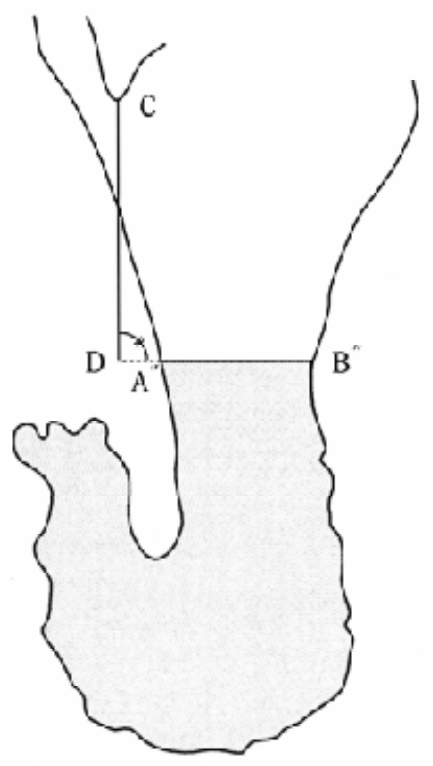

The gray area must be calculated according to the following factors: Dividing line: point A" to point B" Reference line: point $\mathrm{C}$ to point $\mathrm{D}$ Gray area: $120.33 \mathrm{~cm}^{2} ; 114.65 \%$ increased

Fig. 3. continued 


\section{Other features}

(1) Extramural review (independent review of response and of its duration) is desirable.

(2) Factors related to tumor response should be stratified for the evaluation of response.

\section{References}

1. Japanese Research Society for Gastric Cancer. Japanese classification of gastric carcinoma. 1st English ed. Tokyo: Kanehara; 1995.

2. Japanese Gastric Cancer Association. Japanese classification of gastric carcinoma. 13th ed. Tokyo: Kanehara; 1999.

3. Kurihara M, Shimizu H, Tsuboi K, Kobayashi K, Murakami M, Eguchi K, Shimozuma K. Development of quality of life questionnaire in Japan: quality of life assessment of cancer patients receiving chemotherapy. Psychooncology 1999;8:35563.

4. International Conference on Harmonization ( $\mathrm{ICH}$ ) harmonized tripartite guidelines on statistical principles for clinical trials. Full analysis set (5 Feb). 1988. p 5, 21-2. 